\title{
Transfer Function Model Analysis of Two-Phase Traveling Wave Ultrasonic Motor
}

\author{
Dingjie Liu \\ Harbin Institute of Technology, Harbin 150080, P.R. China \\ Idj0904@hotmail.com
}

Keywords: Ultrasonic Motor; System Identification; Speed Model

\begin{abstract}
Considering the difficulty to build a proper model from theoretical derivation, system identification is being applied greatly in practical engineering. In this paper, specific to ultrasonic motor (USM), a practical and effective method of identify the structure and parameter is introduced. By fitting the curve of transient process of USM amplitude step for different orders, an effective method for estimate the transfer function is also introduced. Accordingly, the system with three orders should be applied in engineering. The paper provides a proper method to identify the model of USM. However, in terms of other types of execute components; a large number of experiments are requested as well.
\end{abstract}

\section{Introduction}

Ultrasonic motor is one of the typical representative for newborn solid state motors. The new execute components work like this: Driven by a pair of specific sine-type signal, the piezoceramic, attached to stator tightly, deform and the deformation makes the points of stator be in motion. The motion is amplified by the special friction material and drive the rotor rotate with load. The unique operating principle of USM bring it many unique advantages, comparing with traditional electromagnetic motors: High torque densities (almost 5-10 times as large as electromagnetic motors), low rotating speed(reduction gearbox is not requested), greatly high response speed(several millisecond merely),power-off locked and completely silence(working frequency of a few tens of kilohertzs) Besides, the unique working mechanism makes USM can work in low temperature environment and exterior electric field. These advantages make USM have much potential application value, especially in some special fields like aerospace and intelligent control $[1,2,3]$.

However, owing to its unique operating principle as well, great difficulty of building proper model and designing controller is brought. As a typical nonlinear system, it contains several nonlinear processing like energy conversion between pressure and electric or friction. Accordingly, it is a nonlinear, time-varying and strong-coupling control system with a complex structure. Some paper $[2,4]$ tried to deduce the theoretical model for USM and gained good results. However, except for the complicated mathematical derivation, systems with a high order number cannot be realized in practice. As to methods of building model with system identification, two are applied primarily: frequency modulation and amplitude modulation[5,6]. Frequency modulation, based on the excellent linear characteristic between frequency and steady rotation around a certain working point, cannot predict the transient speed data of USM. Amplitude modulation is requested to predict the transient speed curve.

Based on the theoretical model with seven-order[2], the order number is decreased through the common driving method, and a certain speed curve is fitted with different order number. After gaining transfer functions, the regulation of estimated models is introduced. Finally, the uncertain models with different order number is fitted as well. Through combination of theory and experiment, the optimal structure is determined.

\section{Model Orders Analysis}

Theoretical Model Analysis. Many methods of building model for USM from theoretical derivation have been applied. Some models are built as typical Newton mechanics models, 
containing masses, springs and dampers; some models are built as equivalent circuit models, using the circuit elements like resistance, capacitance and inductance to reveal the speed performance[4]. The model, used for reference in this paper, bases on the kinematic equation and Hamilton Principle[2]:

$$
\left\{\begin{array}{c}
\dot{\mathrm{x}_{\mathrm{s}}} \\
\dot{\mathrm{x}_{\mathrm{r}}}
\end{array}\right\}=\left[\begin{array}{cc}
A_{\mathrm{s}} & 0_{2 \mathrm{n} * 4} \\
0_{4 * 2 \mathrm{n}} & \mathrm{A}_{\mathrm{r}}
\end{array}\right]\left\{\begin{array}{l}
\mathrm{x}_{\mathrm{s}} \\
\mathrm{x}_{\mathrm{r}}
\end{array}\right\}+\left[\begin{array}{cc}
B_{\mathrm{s}} & 0_{2 \mathrm{n} * 2} \\
0_{4 * \mathrm{n}} & B_{\mathrm{r}}
\end{array}\right]\left\{\begin{array}{l}
\mathrm{f}_{\mathrm{s}} \\
\mathrm{f}_{\mathrm{r}}
\end{array}\right\}
$$

$\mathrm{x}_{\mathrm{s}, \mathrm{r}}$ represent the modal amplitude of stator and rotor respectively. $\mathrm{f}_{\mathrm{s}, \mathrm{r}}$ represent the force input matrix for stator and rotor respectively, which is influenced by electric field, normal and tangential force. $A_{s, r}, B_{s, r}$ represent the system matrix and control matrix for stator and rotor respectively, which are only dependent on the interior parameter of USM.

After some analysis and process for the parameter of Eq.1, the state-space equations can be written as follow:

$$
\left[\begin{array}{c}
\dot{\mathrm{x}}_{1} \\
\dot{\mathrm{x}}_{2} \\
\dot{\mathrm{x}}_{3} \\
\dot{\mathrm{x}}_{4} \\
\dot{\mathrm{x}}_{5} \\
\dot{\mathrm{x}}_{6} \\
\dot{\mathrm{x}}_{7}
\end{array}\right]=\left[\begin{array}{ccccccc}
0 & 0 & 1 & 0 & 0 & 0 & 0 \\
0 & 0 & 0 & 1 & 0 & 0 & 0 \\
-\frac{k}{m} & 0 & -\frac{c}{m} & 0 & 0 & 0 & 0 \\
0 & -\frac{k}{m} & 0 & -\frac{c}{m} & 0 & 0 & 0 \\
0 & 0 & 0 & 0 & 0 & 1 & 0 \\
0 & 0 & 0 & 0 & 0 & -\frac{c_{z}}{m_{r}} & 0 \\
0 & 0 & 0 & 0 & 0 & 0 & -\frac{c_{\alpha}}{I_{r}}
\end{array}\right] \cdot\left[\begin{array}{c}
\mathrm{x}_{1} \\
\mathrm{x}_{2} \\
\mathrm{x}_{3} \\
\mathrm{x}_{4} \\
\mathrm{x}_{5} \\
\mathrm{x}_{6} \\
\mathrm{x}_{7}
\end{array}\right]+\left[\begin{array}{ccc}
0 & 0 & 0 \\
0 & 0 & 0 \\
\frac{1}{m} & 0 & 0 \\
\frac{1}{m} & 0 & 0 \\
0 & 0 & 0 \\
0 & \frac{1}{m_{r}} & 0 \\
0 & 0 & 1
\end{array}\right] \cdot\left[\begin{array}{c}
f_{s}(t) \\
F(t) \\
\tau(t)
\end{array}\right]
$$

$\mathrm{x}_{1}-\mathrm{x}_{7}$ represent the $\mathrm{A}$ mechanical modal amplitude, $\mathrm{B}$ mechanical modal amplitude, the differential of A mechanical modal amplitude,B mechanical modal amplitude, flexure height, the differential of flexture height and speed. $k, m, c$ represent the stiffness, mass and damp ratio of USM respectively. Specially, $c_{z}, c_{\alpha}$ represent the rotating damp factor in z-direction and about z-direction respectively. $m_{r}$ represents the mass of rotor. $f_{s}(t), F(t), \tau(t)$ represent the friction force of stator, applied axial load and input torque matrix respectively. In addition, the elements of these three matrix are not only dependent on time but also the modal amplitude, speed and flexure height.

Model Reducing Process. Obviously, the theoretical model with seven orders gained from Eq.2 cannot be applied directly for control system and essential model reducing process is requested. The reducing method bases on a common driving method[3]: Two orthogonal modes(time and space) are applied to generate a traveling wave within the stator, and the mechanical modal amplitudes of $\mathrm{A}$ and $\mathrm{B}$ are equal. Based on the relationship, the mechanical modal amplitudes of $\mathrm{A}$ and $\mathrm{B}$ are not independent variable. Briefly, through knowledge of trigonometric function, the relationships of the mechanical modal amplitudes of $\mathrm{A}$ and $\mathrm{B}$ can be expressed as follow:

$$
\left\{\begin{array}{c}
A \cos (\omega t)=A \omega \sin (\omega t)^{(1)} \\
A \cos (\omega t)^{(1)}=-A \omega \sin (\omega t)
\end{array}\right.
$$

Accordingly,Eq.2 can be processed as follow: 


$$
\left[\begin{array}{c}
\dot{\mathrm{x}}_{1} \\
\dot{\mathrm{x}}_{2} \\
\dot{\mathrm{x}}_{3} \\
\dot{\mathrm{x}_{4}} \\
\dot{\mathrm{x}}_{5}
\end{array}\right]=\left[\begin{array}{ccccc}
0 & 1 & 0 & 0 & 0 \\
-\frac{k}{m} & -\frac{c}{m} & 0 & 0 & 0 \\
0 & 0 & 0 & 1 & 0 \\
0 & 0 & 0 & -\frac{c_{Z}}{m_{r}} & 0 \\
0 & 0 & 0 & 0 & -\frac{c_{\alpha}}{I_{r}}
\end{array}\right] \cdot\left[\begin{array}{c}
\mathrm{x}_{1} \\
\mathrm{x}_{2} \\
\mathrm{x}_{3} \\
\mathrm{x}_{4} \\
\mathrm{x}_{5}
\end{array}\right]+\left[\begin{array}{ccc}
0 & 0 & 0 \\
\frac{1}{m} & 0 & 0 \\
0 & 0 & 0 \\
0 & \frac{1}{m_{r}} & 0 \\
0 & 0 & 1
\end{array}\right] \cdot\left[\begin{array}{c}
f_{S}(t) \\
F(t) \\
\tau(t)
\end{array}\right]
$$

$\mathrm{x}_{1}$ represents the mechanical modal amplitude of a certain electrode pattern.

However, it is also hard to realize the complex control system, as Eq.4 shown. Actually, the systems which are applied in practice have the order numbers from one to three. For some relatively complex systems, some usual methods [7] of designing controller like $H_{\infty}$ controller, $\mu_{\infty}$ controller can be applied. The focus on this paper is to estimate the transfer function models with different orders, considering complexity and veracity of USM system.

\section{Model Identification}

Model Parameter Identification. Design a serial of experiments to gain different transfer function models[8], which are related to voltage amplitude and speed, with USM (Shinsei USR 60-S3). The voltage amplitude is designed at $150 \mathrm{~V}(148.8 \mathrm{~V}$ in measure). Table 1 shows the speed data of transient process driven by voltage amplitude step. The unit of speed is Impulses Per Second (IPS). The steady speed data is about 1150 IPS. unit of speed is Impulses Per Second (IPS).

Table 1 Transient speed data

\begin{tabular}{|l|l|l|l|l|l|l|}
\hline times & 0 & $1(\mathrm{~ms})$ & $2(\mathrm{~ms})$ & $3(\mathrm{~ms})$ & $4(\mathrm{~ms})$ & $5(\mathrm{~ms})$ \\
\hline 1(IPS) & 0 & 544.0 & 810.4 & 948.2 & 953.3 & 1045.1 \\
\hline 2(IPS) & 0 & 539.0 & 733.2 & 915.0 & 1035.5 & 1041.0 \\
\hline 3(IPS) & 0 & 458.0 & 728.8 & 876.9 & 914.6 & 1013.1 \\
\hline 4(IPS) & 0 & 467.4 & 740.5 & 911.4 & 1049.0 & 1044.7 \\
\hline $5($ IPS $)$ & 0 & 614.2 & 811.6 & 965.6 & 1025.6 & 1043.7 \\
\hline Average & 0 & 524.52 & 764.9 & 923.42 & 995.6 & 1037.52 \\
\hline
\end{tabular}

Identify the average data of transient process speed with a serial transfer function structures (order:1 to 3), and comparing with 5 orders curve:

Identification Results (dashed line represents the practical speed curve):As Fig. 1,Fig. 2,Fig. 3 and Fig. 4 shown.

$$
G_{1}(s)=\frac{4172}{s+535.7}
$$




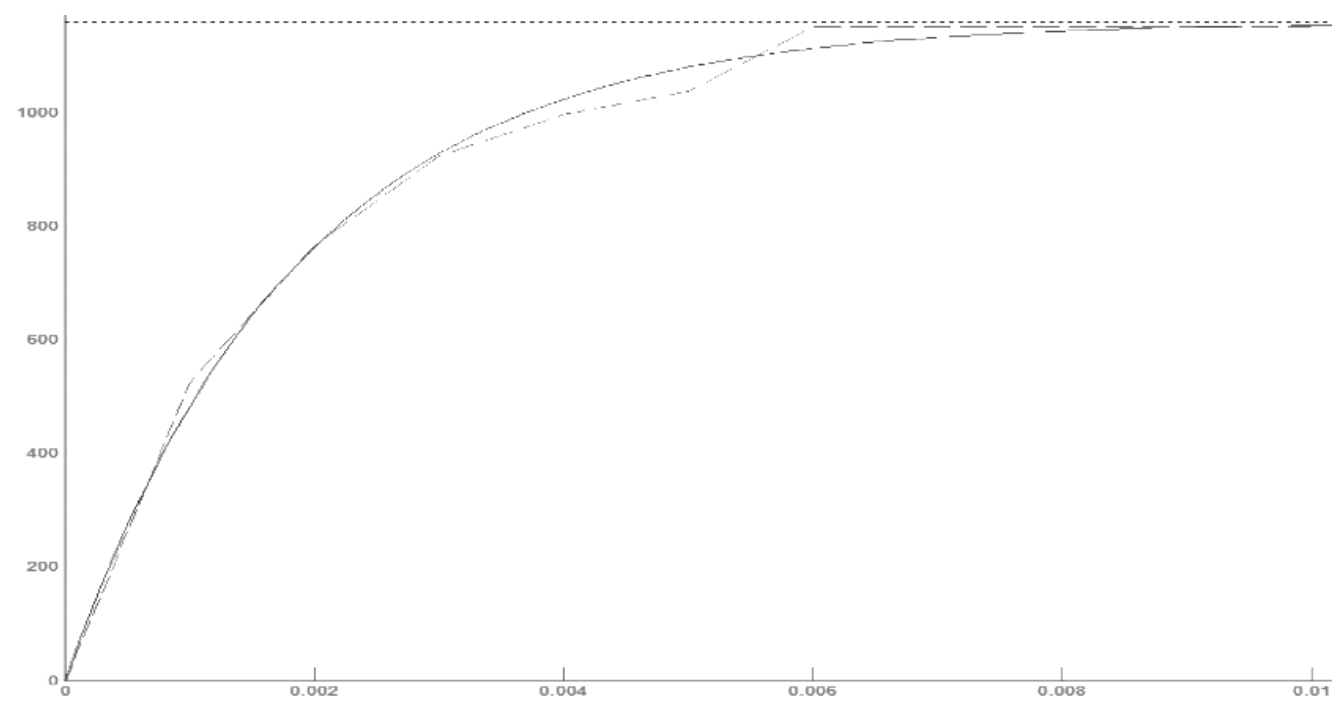

Figure 1. Curve fitted as one order system

$$
G_{2}(s)=\frac{7195 s+1.41 \times 10^{7}}{s^{2}+4378 s+1.785 \times 10^{6}}
$$

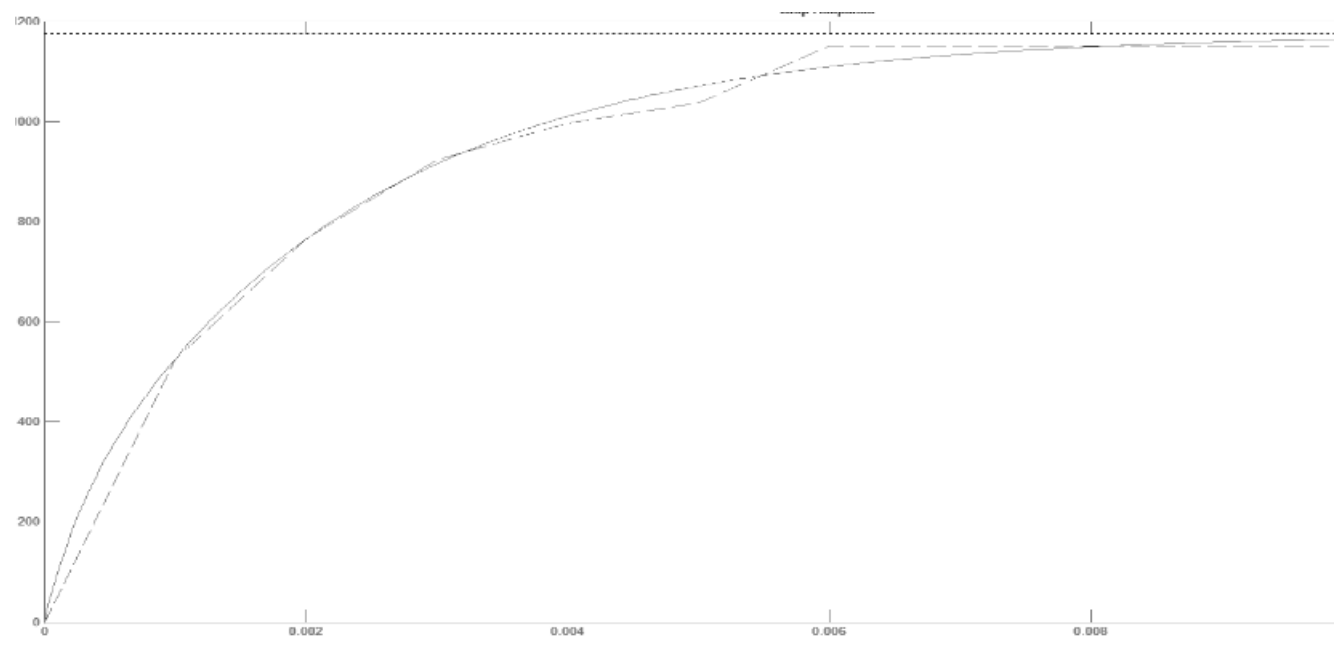

Figure 2. Curve fitted as two order system

$$
G_{3}(s)=\frac{4491 s^{2}+1.354 \times 10^{6} s+5.196 \times 10^{9}}{s^{3}+798.4 s^{2}+1.426 \times 10^{6}+6.622 \times 10^{8}}
$$

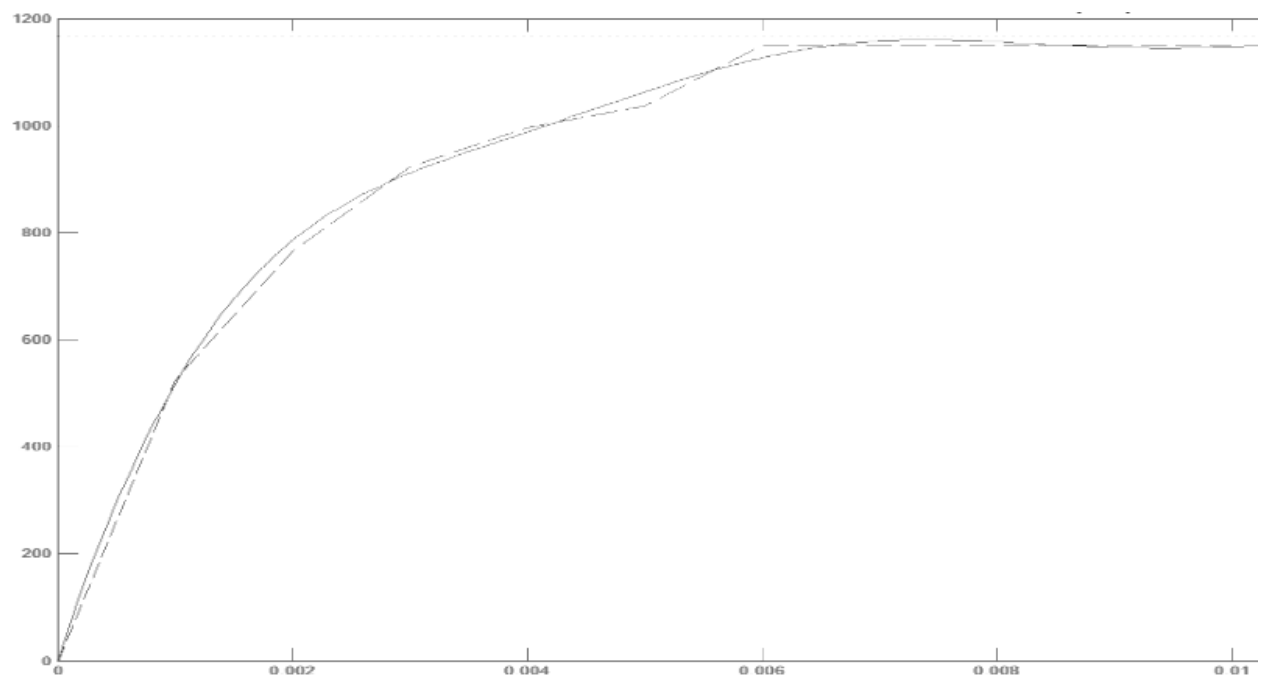

Figure 3. Curve fitted as three order system 


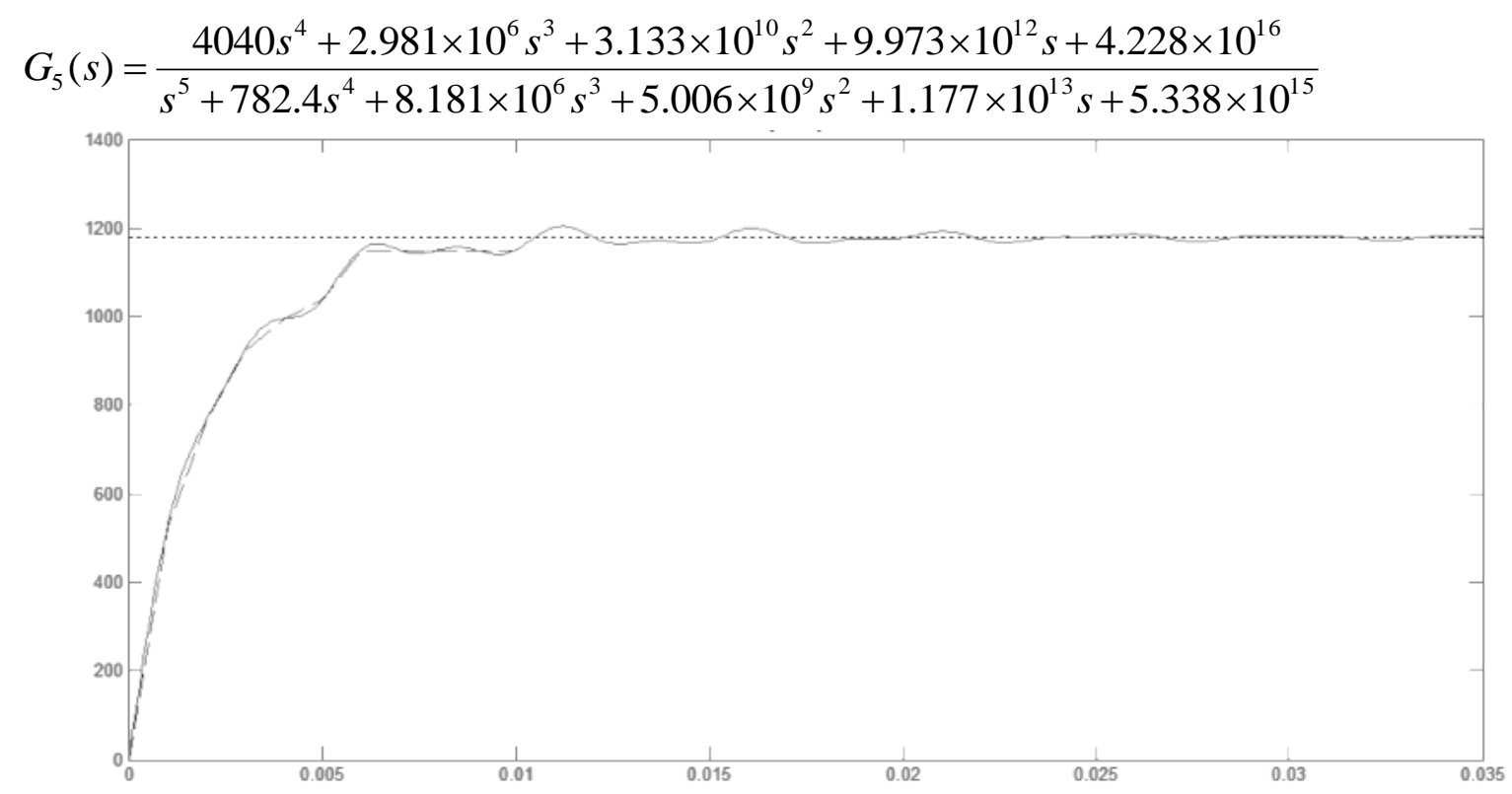

Figure 4. Curve fitted as five order system

Model Structure Analysis. From Fig. 4, the effect of fitting curve with 5-order transfer function structure is excellent. However, the its order is too high to realize itself in practice. Finding the model which has a relatively low order as well as a relatively good fitting effect is the real matter of concern. To estimate the order of systems, regulation as followed is made:

1.Mean Square Error(MSE) between predicted speeds and true ones during transient process.

2.Steady State Error (ess) between predicted steady speed and true one.

3.The complexity of order. 2 .

At time of 1-4 ms, the data predicted by different orders curves, MSE and ess are shown as Table

Table 2 The data predicted by different orders curves, MSE and ess

\begin{tabular}{|l|l|l|l|l|l|l|l|}
\hline & $1 \mathrm{~ms}$ & $2 \mathrm{~ms}$ & $3 \mathrm{~ms}$ & $4 \mathrm{~ms}$ & $5 \mathrm{~ms}$ & MSE & Steady Speed \\
\hline 1 & 484 & 762 & 926 & 1020 & 1080 & 811.364 & 1160 \\
\hline 2 & 528 & 763 & 915 & 1010 & 1070 & 269.785 & 1170 \\
\hline 3 & 517 & 784 & 912 & 988 & 1060 & 222.977 & 1170 \\
\hline 5 (theory) & 527 & 765 & 938 & 996 & 1040 & 45.0094 & 1180 \\
\hline Practice & 524.52 & 764.9 & 923.42 & 995.6 & 1037.52 & - & 1150 \\
\hline
\end{tabular}

As Table 2 shown, the difference of different transfer function for ess is marginal, but in terms of MSE, the system of 1-order is not as valid as that of 2-order or 3-order. Comparing with 3-order system, the complexity of 2-order system is acceptable. As a result, a conclusion could be gained:

To most of USM control systems, system of 2-order could meet the performance index demand.

USM Speed Performance of Driving Frequency. As introduction illustrated, a model based on frequency and steady speed is also essential in practice for its excellent effect to predict steady speed. Design an experiment as follow:

The steady speed of USM(Shinsei USR 60-S3) is observed under the decrease of frequency by $100 \mathrm{~Hz}$ (from $42.5 \mathrm{kHz}$ to $40.8 \mathrm{kHz}$ ), whose data is shown on Table 3,Fig. 5: 
Table 3 Steady speed data and frequency

\begin{tabular}{|c|c|c|c|c|c|c|c|}
\hline Items & \multicolumn{7}{|c|}{ data } \\
\hline $\mathrm{Feq} / \mathrm{Hz}$ & 42500 & 42400 & 42300 & 42200 & 42100 & 42000 & 41900 \\
\hline $\begin{array}{c}\text { Speed/ } \\
\text { rpm }\end{array}$ & 68.262 & 72.872 & 78.090 & 82.628 & 87.187 & 92.737 & 97.464 \\
\hline $\mathrm{Feq} / \mathrm{Hz}$ & 41800 & 41700 & 41600 & 41500 & 41400 & 41300 & 41200 \\
\hline $\begin{array}{c}\text { Speed/ } \\
\text { rpm }\end{array}$ & 102.563 & 108.006 & 113.418 & 120.377 & 126.306 & 132.423 & 138.269 \\
\hline $\mathrm{Feq} / \mathrm{Hz}$ & 41100 & 41000 & 40900 & 40800 & & & \\
\hline $\begin{array}{c}\text { Speed/ } \\
\text { rpm }\end{array}$ & 144.010 & 148.790 & 152.441 & 155.621 & & & \\
\hline
\end{tabular}

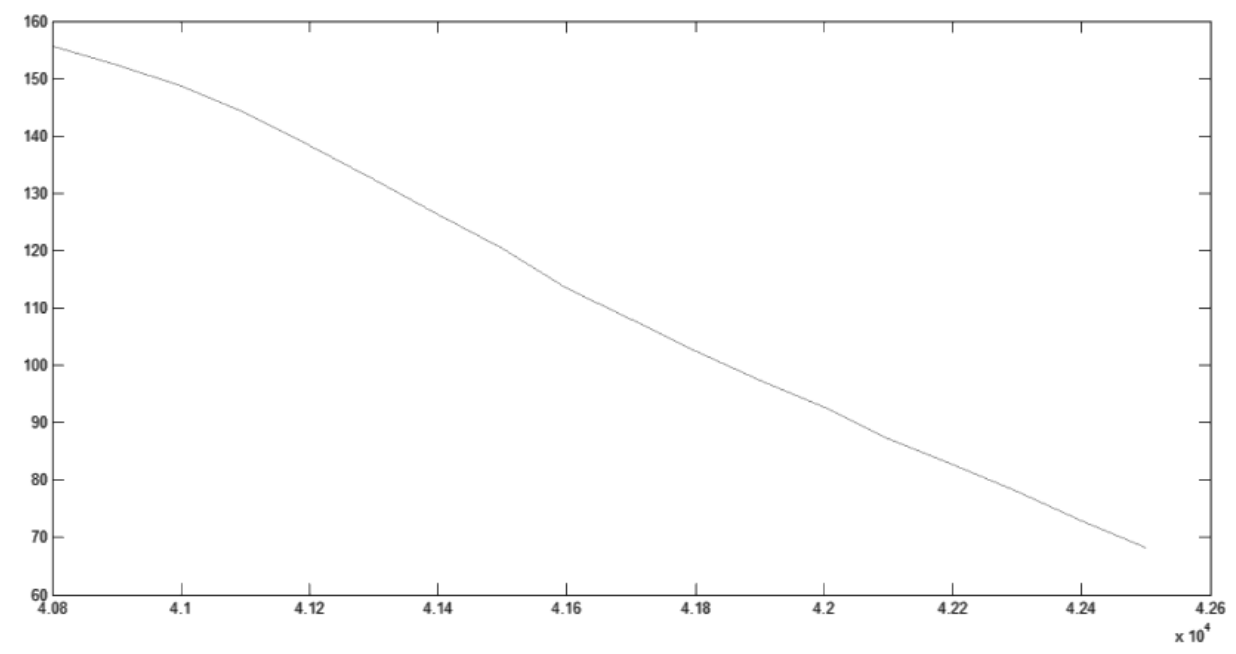

Figure 5. Curve of frequency and steady speed

As observed from Fig. 5 shown, the curve shows an excellent linear relation between frequency and steady speed.

\section{The Uncertain Models Identification}

Uncertainty [7,9]is pretty common in practical engineering. Nominal model can not be applied into practice directly. For applying transfer function model and design robust controller suitably, the uncertain model is requested. Processing the raw data gained from experiments above, the uncertainty bound is gained:

Table 4 Raw speed data

\begin{tabular}{|l|l|c|c|c|c|c|}
\hline E1.Speed/IPS & 0 & 544.0 & 810.4 & 948.2 & 953.3 & 1045.1 \\
\hline E2.Speed/IPS & 0 & 539.0 & 733.2 & 915.0 & 1035.5 & 1041.0 \\
\hline E3.Speed/IPS & 0 & 458.0 & 728.8 & 876.9 & 914.6 & 1013.1 \\
\hline E4.Speed/IPS & 0 & 467.4 & 740.5 & 911.4 & 1049.0 & 1044.7 \\
\hline E5.Speed/IPS & 0 & 614.2 & 811.6 & 965.6 & 1025.6 & 1043.7 \\
\hline
\end{tabular}

After calculation:

1-order: 


$$
\begin{aligned}
& G_{1}(s)=G_{01}(s)+\Delta G_{1}(s)=\frac{k_{1}}{s+a_{1}}: \\
& k_{1} \in[4027,4428], \quad a_{1} \in[511.7,573.4]
\end{aligned}
$$

2-order:

$$
\begin{aligned}
& G_{2}(s)=G_{02}(s)+\Delta G_{2}(s)=\frac{z_{1} s+k_{2}}{s^{2}+a_{2} s+b_{2}}: \\
& z_{1} \in[5443,9024], \quad k_{2} \in\left[4.011 \times 10^{6}, 1.748 \times 10^{7}\right] \\
& a_{2} \in[1740,9505], \quad b_{2} \in\left[4.385 \times 10^{5}, 6.711 \times 10^{6}\right]
\end{aligned}
$$

3-order:

$$
\begin{aligned}
& G_{3}(s)=G_{03}(s)+\Delta G_{3}(s)=\frac{z_{1} s^{2}+z_{2} s+k_{3}}{s^{3}+a_{3} s^{2}+b_{3} s+c_{3}}: \\
& z_{1} \in[3559,4646], \quad z_{2} \in\left[1.107 \times 10^{6}, 2.532 \times 10^{6}\right], \quad k_{3} \in\left[4.514 \times 10^{9}, 6.34 \times 10^{9}\right] \\
& a_{3} \in[551.5,859], \quad b_{3} \in\left[1.006 \times 10^{6}, 2.78 \times 10^{6}\right], \quad b_{2} \in\left[4.943 \times 10^{8}, 8.159 \times 10^{8}\right]
\end{aligned}
$$

\section{Summary}

In this paper, the theoretical model is simplified and the order is determined through the common driving signal characteristic. A serial of transfer function models are fitted, simulated and calculated, based on one speed curve.By estimating the order, the conclusion is made that 2-order system fitting is optimal for most USM control system.Moreover, the model between frequency and steady speed is shown and analyzed as well and the uncertain models are gained.

\section{References}

[1] T. Saqhida, T. Kenjo.An introduction to ultrasonic motors.Clarendon Press-Oxford,1993

[2] Nesbitt W. Hagood IV and Andrew J. McFarland.Modeling of a Piezoelectric Rotary Ultrasonic Motor.IEEE Transactions on ultrasonics, ferroelectrics, and frequency control,1995,42,(2):210-223.

[3] Chunsheng Zhao.The technology and application of ultrasonic motor.Science Press-Beijing,2007

[4] J. Maas, P. Ide, N. Frohleke, H. Grotstollen.Simulation Model for Ultrasonic Motors powered by Resonant Converters.Rec IEEE-IAS Annu. Meeting, vol.1 ,Lake Buena Vista, FL,Oct.1995,pp.111-120.

[5] Furong Lei,Yisheng Zhong,Wenli Xu.Robust tracking control of ultrasonic motors based on interval system description.Proceedings of the CSEE,2004,vol24(1):87-91.

[6] Bo Liu,Jingzhuo Shi. Building frequency-speed model of ultrasonic motors based on step response.Micromotors,2010,vol43(11):77-80.

[7] Kangzhi Liu,Yu Yao.Linear robust control. Science Press-Beijing,2007.

[8] Qinchao Xu,Jiyang Zhang.A type of nonlinear control model of traveling wave ultrasonic motors.Aerospace Control and Application,2008,vol34(4):60-63.

[9] Furong Lei,Yisheng Zhong,Wenli Xu.Robust tracking control of ultrasonic motors based on nonlinear system description.Piezoelectrics \& Acoustooptics,2005,vol27(1):68-73.

[10]Jingzhuo Shi,Lin Lv.Dynamic fuzzy modeling of ultrasonic motors speed control.Proceedings of the CSEE,2011,vol31(33):109-114. 